\title{
Pesearch Square \\ Clinical Factors and Pre- Surgery Depression Scores Predict Pain Intensity in Cardiac Surgery Patients
}

Jacob Gohari

Technion-Israel Institute of Technology

Liza Grosman-Rimon

Poriya Medical Center

Mattan Arazi

Technion-Israel Institute of Technology

Noa Caspi-Avissar

Poriya Medical Center

Dina Granot

Poriya Medical Center

Sagi Gleitman

Poriya Medical Center

Jawdat Badarny BSn

Poriya Medical Center

Alla Lubovich

Poriya Medical Center

Doron Sudarsky

Poriya Medical Center

Shemy Carasso

Poriya Medical Center

\section{Edo Y. Birati}

Technion-Israel Institute of Technology

Erez Kachel ( $\sim$ Erez.Kachel@sheba.health.gov.il)

Poriya Medical Center

\section{Research Article}

Keywords: Cardiac Surgery, Pain, Depression

Posted Date: June 9th, 2021

DOI: https://doi.org/10.21203/rs.3.rs-561564/v1 
License: (c) (i) This work is licensed under a Creative Commons Attribution 4.0 International License. Read Full License 


\section{Abstract}

Objectives: Moderate to severe pain is prevalent in cardiac surgery patients and can increase cardiac complications, morbidity and mortality. The objectives of the study were to assess perioperative pain intensity and to assess predictors of pain post-cardiac surgery, including clinical characteristics and depression.

Methods: A total of 98 cardiac surgery patients were included in the study. Pain intensity was assessed using a Numerical Rating System at 1 day pre-operatively and 2 day post-operatively until hospital discharge. Clinical data were recorded and depression scores were assessed using the Center for Epidemiological Study of Depression (CES-D).

Results: Pain intensity increased significantly during hospitalization from pre-operative levels, surging at 2 day post-operatively. Predictors of high pain intensity were high pre-operative CES-D scores, female gender, cardiac function, smoking and high body mass index (BMI). Significantly higher pre-operative CES-D scores were found in patients with severe pain compared to patients with no pain to moderate pain (18.23 \pm 1.80 vs $12.84 \pm 1.22, p=0.01$ pre-operatively). Patients with severe pain (NRS 7-10) had significantly higher levels of white blood cells (WBC) compared to patients with no pain-moderate pain (NRS 0-6), $(p=0.01)$. However, CES-D scores were only weakly correlated maximum WBC levels perioperatively.

Discussion: Pain intensity significantly increased following surgery, and was associated with depressive symptoms, female sex, cardiac function, BMI, and smoking. These factors may serve as a basis for identification and intervention to help prevent the transition from acute pain to chronic pain.

\section{Introduction}

Post- cardiac surgery depression is prevalent ${ }^{1}$, and is associated with mortality. ${ }^{2,3}$ An increase in depressive symptoms after cardiac surgery is also associated with the occurrence of new cardiac complications. ${ }^{4}$ Accumulating evidence has found that depression is associated with somatic symptoms, especially with pain severity. ${ }^{5}$ Interestingly, up to two-thirds of patients with depression also had moderate to severe pain. ${ }^{6}$ Moreover, one-third of the patients noted persistent or worsening depressive symptoms at post-discharge, with over two-thirds of these patients having pain interfering with daily living. ${ }^{7}$ Post cardiac surgery pain is reported in up to $85 \%$ of patients, with moderate to severe pain occurring usually in the first 2-4 days post-operatively. ${ }^{8}$ Moreover, studies have shown that $39 \%$ of patients post-cardiac surgery have persisting, chronic pain over 2 years post-operation. ${ }^{9}$

Perioperative pain is also a major risk factor which can lead to the development of chronic pain, increased cardiac complications, increased morbidity and increased mortality. ${ }^{10-13}$ 
However, current data is limited on the coexistence of both pain and depressive symptoms in cardiac surgery patients. ${ }^{6,7}$ Moreover, it is not well established whether pre- and post-operative depression has been associated with intensity of post-operative pain in cardiac surgery patients.

The objectives of the current study are to assess the peri-operative pain intensity, and to determine whether perioperative depression scores and clinical characteristics are predictive of post-surgical pain intensity.

\section{Research Methodology}

\section{Study Design}

A prospective study was conducted to investigate the relationship between reported pain scores and depressive symptoms throughout the perioperative period. Pain was measured one day pre-operatively and during 7 days post-operatively using a Numerical Rating System throughout hospitalization. Depression scores were assessed using the Center for Epidemiological Study of Depression (CES-D) at pre-operative, during hospitalization, and up to 10 days post-discharge.

\section{Patient Cohort}

Adult patients undergoing cardiac surgery (valvular surgery or coronary artery bypass grafting) at the Baruch Padeh Poriya Medical Center from March 2018 to June 2020 were included in this study. Patients with chronic inflammatory disease, end-stage renal disease requiring dialysis and patients with evidence of peripheral neuropathy or on chronic pain medication were excluded from the study.

The study was approved by the institutional review board. Signed informed consents were obtained from all study participants. Inclusion Criteria: 1) patients eligible for open-heart surgery (valve surgery or coronary artery bypass grafting (CABG) 2) patients ages 20 and older.

Exclusion Criteria: 1) Patients under the age of 20;2) patients with end-stage renal disease requiring dialysis; 3) presence of peripheral neuropathy; Glasgow Coma Scale score < 13) patient on chronic pain medication; and 5) patients with chronic inflammatory disease were excluded from the study.

\section{Procedures}

Clinical and surgical data were recorded from the electronic medical records. The following variables were recorded: Age, BMI, left ventricle ejection fraction (EF) less than $50 \%$, white blood cell count, Working status, Smoking status, Diabetes, Residence, Education level, Marital status, Numeric Pain Rating, and CES-D depression scores

\section{Assessment of Pain}

Pain was measured using a Numerical Rating System (NRS), which is an 11 point interval scale evaluating pain from 0 -to- 10 , with 0 characterizing no pain and 10 characterizing maximum pain. ${ }^{14,15}$ 
Pain severity was divided into 4 categories based on the NRS scores: no pain (score of 0), mild pain (score of 1-3), moderate pain (score of 4-6), and severe pain (score of 7-10). ${ }^{15}$ Assessment of Depression

The Center for Epidemiological Study of Depression (CES-D) scale was used to measure depression scores in patients, as it has demonstrated acceptable reliability amongst various patient groups. ${ }^{16}$ The 20 item, self-reported questionnaire uses a four-point Likert scale to measure depressive symptoms, with scores ranging from 0 (lowest) to 60 (highest). Depression scores were divided into 3 levels: no depression (level-1, CES-D < 15); mild depression (level-2, CES-D between 16 and 26); and moderate to severe (level-3, CES-D > 27) depression, and prevalence at each time point was calculated.

\section{Statistical Analysis}

Statistical analysis was performed using SPSS (Version 22.0. Armonk, NY: IBM Corp). Data was reported as mean \pm SE or as percentages. One-way repeated measure analysis of variance (ANOVA) was performed to compare the pain intensity longitudinally across assessment time points. Pain severity was divided into 4 categories based on the NRS scores: no pain (score of 0 ), mild pain (score of 1-3), moderate pain (score of 4-6), and severe pain (score of 7-10). The rate of pain (calculated as the number of patients with pain divided by the total number patients) and the percentage of patients with pain (calculated by multiplying the rate by 100 ) was calculated across assessment time points. One-way repeated measure analysis of variance (ANOVA) was performed to compare the depression scores longitudinally across assessment time points. Independent T-test analysis was performed to compare the CES-D scores at pre-operative, hospitalization, and 10 days post-discharge time points, between patients with no pain-to-moderate pain (NRS score of $0-6$ ) and severe pain (NRS score of 7-10) post surgery.

A univariate logistic regression was performed to examine the association between pain intensity category (severe pain or no pain-moderate pain) and the following variables: pre-operative CES-D, hospitalization CES-D, CES-D 10 day post-discharge, age, sex, diabetes, ejection fraction, BMI, and smoking status. Multivariable logistic regressions were performed forcing all covariates with statistical significance of $<0.175$ into the model. Backward variable elimination was used to develop the regression model. Those variables with a statistically significant level of $<0.1$ were retained in the final model. In addition, the differences in depression scores between patients with severe pain and patients with no pain-moderate pain were examined using an independent-samples t-test. A p-value of $<0.05$ was considered significant.

A 2-way repeated measure ANOVA was performed to assess levels of WBC longitudinally, comparing the 2 pain categories: no pain-moderate pain (NRS of 0-6) and severe pain (NRS 7-10) A p-value of $<0.05$ was considered significant. The association between pain scores, CES-D scores, and WBC levels were assessed at pre-operative and post-operative time points using the Pearson correlation coefficient.

\section{Results}


A total of 98 patients were included in this study. The mean age was 61 years old and there were $25 \%$ female patients. In this patient's cohort, $36 \%$ of the patients were diagnosed with diabetes and the average BMI was $25.5 \mathrm{~kg} / \mathrm{m}^{2} .70 \%$ of the patients had preserved ejection fraction (left ventricle EF higher than $50 \%$ ) and $40 \%$ were active smokers. Baseline characteristics in patients with No pain- Moderate pain and patients with severe pain are presented in Table 1.

Table 1

Baseline characteristics in patients with No pain- Moderate pain compared to patients Severe Pain

\begin{tabular}{|c|c|c|c|}
\hline & $\begin{array}{l}\text { No pain- Moderate pain (NRS 0-6) } \\
(n=55)\end{array}$ & $\begin{array}{l}\text { Severe Pain } \\
\text { (NRS 7-10) } \\
(n=43)\end{array}$ & p-value \\
\hline Age (years) & $62.4 \pm 8.6$ & $58.8 \pm 9.7$ & 0.63 \\
\hline $\mathrm{BMI}\left(\mathrm{kg} / \mathrm{m}^{2}\right)$ & $27.5 \pm 4.0$ & $29.44 \pm 4.2$ & 0.30 \\
\hline Sex (female) \% (n) & $94.6 \%(n=53)$ & $70.5 \%(n=31)$ & 0.01 \\
\hline Diabetes \% (n) & $30.4 \%(n=17)$ & $45.5 \%(n=20)$ & 0.21 \\
\hline $\mathrm{EF}<50 \%(\mathrm{n})$ & $37.5 \%(n=21)$ & $22.7 \%(n=10)$ & 0.11 \\
\hline Non Smoking \% (n) & $32.1 \%(n=18)$ & $45.5 \%(n=20)$ & 0.17 \\
\hline
\end{tabular}

Pain intensity increased significantly during hospitalization from pre-operative levels, peaking at postoperative day (POD) $2(p<0.0001)$. Pain intensity gradually decreased during the first week postoperatively 7 days of follow-up, but remained higher than the baseline levels $(p<0.0001)$. Although the percentage of patients with pain decreased gradually during the first week, the percentages of patients reporting moderate (NRS 4-6), and severe (NRS 7-10) pain remained high, with $83 \%$ of patients reporting moderate-severe pain on POD-2, $60 \%$ on POD-3, $41 \%$ on post-operative day- $4,27 \%$ on postoperative day- $5,22 \%$ on post-operative day- 6 , and $19 \%$ post-operative day-7 (Fig. 1).

A significant higher pre-operatively CES-D scores was found in patients with severe (NRS 7-10) postoperative pain compared to patients with no pain to moderate pain (NRS 0-6) (Fig. 2) (18.23 $\pm 1.80 \mathrm{vs}$ $12.84 \pm 1.22, p=0.01)$. There was a trend of higher CES-D scores of patients with severe pain compared to patients with no pain-moderate pain during hospitalization ( $22.60 \pm 1.46$ vs. $20.77 \pm 1.36)$ and 10 -days post discharge $(17.30 \pm 1.72$ vs. $15.38 \pm 1.27)$, but the levels did not reach significance.

A Pearson correlation indicates that pre-operative CES-D scores was weakly correlated with maximum pain intensity $(r=0.2, p=0.049)$. Hospitalization and 10 days post-discharge CES-D scores were also weakly correlated with maximum white blood cell count perioperatively $(r=0.229, p=0.022$ and $r=0.213$, $p=0.035$, respectively). 
A 2-way repeated measure ANOVA revealed that patients with severe pain (NRS 7-10) had significantly higher levels of WBC compared to patients with no to moderate pain (NRS $0-6),(p=0.01)$ (Fig. 3). In both groups, the levels of WBC increased from pre-operative levels and peaked at day 2 post-operatively, followed by a gradual decrease in WBC levels.

A univariate analysis revealed that pre-operative CES-D, female sex, and high BMI were significant predictors of pain intensity during hospitalization (Table 2 ). In a multivariate regression, pre-operative CES-D, female sex, reduced left ventricle $\mathrm{EF}(<50 \%), \mathrm{BMI}$, and smoking were associated with increased maximal pain scores (Nagelkerke $R^{2}=0.40, \chi^{2}=35.1 \mathrm{p}<0.001$ ).

Table 2

Univariable and Multivariable Analysis of Independent Predictors of Post-operative Pain Intensity

\begin{tabular}{|lllll|}
\hline & Univariable Analysis & \multicolumn{3}{l|}{ Multivariable Analysis } \\
\hline Parameter & OR $(95 \% \mathrm{Cl})$ & $\begin{array}{l}\text { P- } \\
\text { value }\end{array}$ & OR $(95 \% \mathrm{Cl})$ & P-value \\
\hline Age & $0.958(0.916-1.003)$ & 0.067 & - & - \\
\hline Sex & $7.409(1.957-$ & 0.003 & $13.733(2.676-$ & 0.002 \\
\hline BMl & $28.049)$ & & $70.485)$ & 0.020 \\
\hline Pre-op CES-D & $1.114(1.008-1.232)$ & 0.034 & $1.148(1.022-1.291)$ & 0.023 \\
\hline Hospitalization CES-D & $1.050(1.009-1.093)$ & 0.016 & $1.059(1.008-1.113)$ & - \\
\hline 10 Day Post-Discharge CES- & $1.019(.979-1.061)$ & 0.360 & - & - \\
\hline D & $1.019(.980-1.059)$ & 0.355 & - & - \\
\hline Diabetes & $0.523(0.23-1.191)$ & 0.123 & - & 0.011 \\
\hline Ejection Fraction & $2.050(0.839-4.961)$ & 0.116 & $4.259(1.388-13.068)$ & \\
\hline Non Smoking & $0.568(0.251-1.286)$ & 0.175 & $0.316(0.111-0.897)$ & 0.030 \\
\hline
\end{tabular}

\section{Discussion}

The main finding of the study is that patients with severe post-surgical pain had significantly higher depression scores pre-operatively than patients with no pain to moderate pain.

In our study, predictors of post-cardiac surgery pain intensity were pre-operative CES-D scores, smoking, high BMI, female sex, and ejection fraction $(<50 \%)$. The majority of these factors are modifiable. Studies have reported that cardiac surgery patients pain is more prevalent in depressive symptoms. ${ }^{6,7}$ Since uncontrolled pain post-cardiac surgery increases morbidity risk, preoperative depression screening may identify which patients require increased care following surgery. ${ }^{11,13}$ In addition, it is possible that preoperative treatments of depression may reduce risk of developing severe pain post-cardiac surgery. 
Furthermore, interventions aiming at lifestyle modification, such as smoking cessation during the preoperative period that were shown to be beneficial for many clinical outcomes ${ }^{17,18}$, may also be beneficial for pain management. In addition, exercise training was reported have beneficial effects on depression ${ }^{19}$. Patients who exercised prior to the surgery may experience less depression symptoms and lower pain intensity.

In our patient population, pain peaked post-operatively, which is consistent with current literature. ${ }^{8,20}$ In fact, uncontrolled pain post-cardiac surgery increases morbidity risk. ${ }^{11,13}$ Our findings that women experienced higher pain intensity is consistent with previous literature examining post-surgical pain, with previous studies observing a higher rate of pain in women. ${ }^{21,22}$ Studies have also noted a greater frequency of persistent pain at 12 months post-cardiac surgery in women compared to men. ${ }^{23}$ Our study observed no significant association between CES-D scores during hospitalization and maximum pain intensity. However, we found a significant association between maximum pain intensity during hospitalization and pre-operative CES-D levels. One reason for the lack of significant association between pain intensity and CES-D scores during hospitalization may be due to the use of multiple pain medications being used throughout the hospitalization period, which may have affected each patient's subjective pain scores. Interestingly, we found that prior to surgery, $1 \%$ of our cohort experienced preoperative pain. Pre-operative pain is common, with $38 \%$ of cardiac surgery patients reporting pain prior to surgery. ${ }^{8}$ Further research should investigate whether treatment of pain prior to surgery may affect postoperative outcomes, including subjective pain symptoms. With anti-depressants typically taking a few weeks for clinical effects to become apparent, pre-operative treatment with a selective serotonin reuptake inhibitor (SSRI) or selective norepinephrine reuptake inhibitor (SNRI) with the addition of antiinflammatory medications post-operatively may be an option to be further investigated.

Post-surgical pain may also be linked not only to depressive symptoms, but also to the trauma associated with the procedure and the elevated white blood cell (WBC) levels induced by the surgery. ${ }^{24,25}$ In this study, we evaluated the levels of WBC, since their levels may be related to the trauma associated with the surgical procedure. ${ }^{24,25}$ Our study found a significant association between WBC levels and pain intensity levels throughout the hospitalization period. However. Our findings of an association between CES-D scores and increased WBC levels are consistent with a recent meta-analysis which noted that major depressive disorder is associated with overall leukocytosis. ${ }^{26}$ Future studies should examine whether patients in this population may benefit from a multi-pronged approach targeting pain and depression.

\section{Conclusion}

Pain intensity significantly increased post-cardiac surgery, and is associated with depressive symptoms, female sex, cardiac function, BMl, and smoking. These factors may serve as a basis for identification and intervention to help prevent the transition from acute pain to chronic pain. Moreover, physicians should anticipate higher levels of pain in patients with higher CES-D scores. The high depressive scores found 
post-operatively highlight the need to establish depression screening as part of the pre-operative patient management. Future research should investigate the effectiveness of post-cardiac surgery pain treatments and determine whether perioperative treatment with antidepressants medications can affect patient outcomes.

\section{Abbreviations}

CES-D, center for Epidemiological Study of Depression

WBC, white blood cells

BMI, body mass index

CABG, coronary artery bypass grafting

EF, ejection fraction

NRS, numerical Rating System

ANOVA, analysis of variance

POD, post-operative day

SSRI, a selective serotonin reuptake inhibitor

SNRI, selective norepinephrine reuptake inhibitor

\section{Declarations}

\section{Ethics approval and consent to participate}

The study protocol was reviewed and approved by the Poriya Medical Center's Ethical Review Board. Informed consent was obtained from each patient prior to participating in the study. The research study was conducted in accordance with the World Medical Association Declaration of Helsinki.

\section{Consent for Publication}

'Not applicable

\section{Availability of data and material}

The data is available upon request from the corresponding author.

\section{Competing interests}

The authors have no competing interests to disclose. 


\section{Funding}

No funding was provided to support this study.

\section{Authors' contributions}

JG wrote the manuscript; LGR analyzed the data, interpreted the findings, wrote the manuscript and approved the final version; MA edited the manuscript; NCA collected the data and interpreted the findings; DG collected the data and interpreted the findings; SG interpreted the findings and edited the manuscript; JB collected the data and interpreted the findings; AL edited the manuscript, DS edited the manuscript; SC designed the study, edited the manuscript and approved the final version; EYB designed the study and edited the manuscript and approved the final version; EK designed the study and edited the manuscript and approved the final version.

\section{Acknowledgements}

The authors wise to thank the participants of the study.

\section{References}

1. Caspi-Avissar N, Grosman-Rimon L, Gohari J, et al. Clinical, Surgical, and Socio-Psychological Factors and Depression Post-Cardiothoracic Surgery. Ann Thorac Surg. 2020.

2. Tully PJ, Baker RA. Depression, anxiety, and cardiac morbidity outcomes after coronary artery bypass surgery: a contemporary and practical review. Journal of geriatric cardiology: JGC. 2012;9(2):197208.

3. Blumenthal JA, Lett HS, Babyak MA, et al. Depression as a risk factor for mortality after coronary artery bypass surgery. Lancet. 2003;362(9384):604-609.

4. Peterson JC, Charlson ME, Williams-Russo $P$, et al. New postoperative depressive symptoms and long-term cardiac outcomes after coronary artery bypass surgery. The American journal of geriatric psychiatry: official journal of the American Association for Geriatric Psychiatry. 2002;10(2):192-198.

5. Novick D, Montgomery W, Aguado J, et al. Which somatic symptoms are associated with an unfavorable course in Asian patients with major depressive disorder? Journal of affective disorders. 2013;149(1-3):182-188.

6. Morone NE, Weiner DK, Belnap BH, et al. The impact of pain and depression on recovery after coronary artery bypass grafting. Psychosomatic medicine. 2010;72(7):620-625.

7. Doering LV, Chen B, McGuire A, Bodán RC, Irwin MR. Persistent depressive symptoms and pain after cardiac surgery. Psychosomatic medicine. 2014;76(6):437-444.

8. Bjørnnes AK, Rustøen T, Lie I, Watt-Watson J, Leegaard M. Pain characteristics and analgesic intake before and following cardiac surgery. European journal of cardiovascular nursing: journal of the Working Group on Cardiovascular Nursing of the European Society of Cardiology. 2016;15(1):47-54. 
9. Bruce J, Drury N, Poobalan AS, Jeffrey RR, Smith WC, Chambers WA. The prevalence of chronic chest and leg pain following cardiac surgery: a historical cohort study. Pain. 2003;104(1-2):265-273.

10. Kehlet $\mathrm{H}$, Jensen TS, Woolf CJ. Persistent postsurgical pain: risk factors and prevention. Lancet. 2006;367(9522):1618-1625.

11. Katz J, Seltzer Z. Transition from acute to chronic postsurgical pain: risk factors and protective factors. Expert review of neurotherapeutics. 2009;9(5):723-744.

12. Rodgers A, Walker N, Schug S, et al. Reduction of postoperative mortality and morbidity with epidural or spinal anaesthesia: results from overview of randomised trials. Bmj. 2000;321(7275):1493.

13. Wu CL, Raja SN. Treatment of acute postoperative pain. Lancet. 2011;377(9784):2215-2225.

14. Zubrzycki M, Liebold A, Skrabal C, et al. Assessment and pathophysiology of pain in cardiac surgery. Journal of pain research. 2018;11:1599-1611.

15. Breivik H, Borchgrevink PC, Allen SM, et al. Assessment of pain. British journal of anaesthesia. 2008;101(1):17-24.

16. Vilagut G, Forero CG, Barbaglia G, Alonso J. Screening for Depression in the General Population with the Center for Epidemiologic Studies Depression (CES-D): A Systematic Review with Meta-Analysis. PLoS One. 2016;11(5):e0155431.

17. van Domburg RT, Meeter K, van Berkel DF, Veldkamp RF, van Herwerden LA, Bogers AJ. Smoking cessation reduces mortality after coronary artery bypass surgery: a 20-year follow-up study. J Am Coll Cardiol. 2000;36(3):878-883.

18. Sørensen LT. Wound healing and infection in surgery. The clinical impact of smoking and smoking cessation: a systematic review and meta-analysis. Archives of surgery (Chicago, III: 1960). 2012;147(4):373-383.

19. Blumenthal JA, Babyak MA, Moore KA, et al. Effects of exercise training on older patients with major depression. Arch Intern Med. 1999;159(19):2349-2356.

20. Gerbershagen HJ, Aduckathil S, van Wijck AJ, Peelen LM, Kalkman CJ, Meissner W. Pain intensity on the first day after surgery: a prospective cohort study comparing 179 surgical procedures. Anesthesiology. 2013;118(4):934-944.

21. Vallerand AH, Polomano RC. The relationship of gender to pain. Pain management nursing: official journal of the American Society of Pain Management Nurses. 2000;1(3 Suppl 1):8-15.

22. Rosseland LA, Stubhaug A. Gender is a confounding factor in pain trials: women report more pain than men after arthroscopic surgery. Pain. 2004;112(3):248-253.

23. Bjørnnes AK, Parry M, Lie I, et al. Pain experiences of men and women after cardiac surgery. J Clin Nurs. 2016;25(19-20):3058-3068.

24. Paladino L, Subramanian RA, Bonilla E, Sinert RH. Leukocytosis as prognostic indicator of major injury. The western journal of emergency medicine. 2010;11(5):450-455.

25. Santucci CA, Purcell TB, Mejia C. Leukocytosis as a predictor of severe injury in blunt trauma. The western journal of emergency medicine. 2008;9(2):81-85. 
26. Zorrilla EP, Luborsky L, McKay JR, et al. The relationship of depression and stressors to immunological assays: a meta-analytic review. Brain Behav Immun. 2001;15(3):199-226.

\section{Figures}

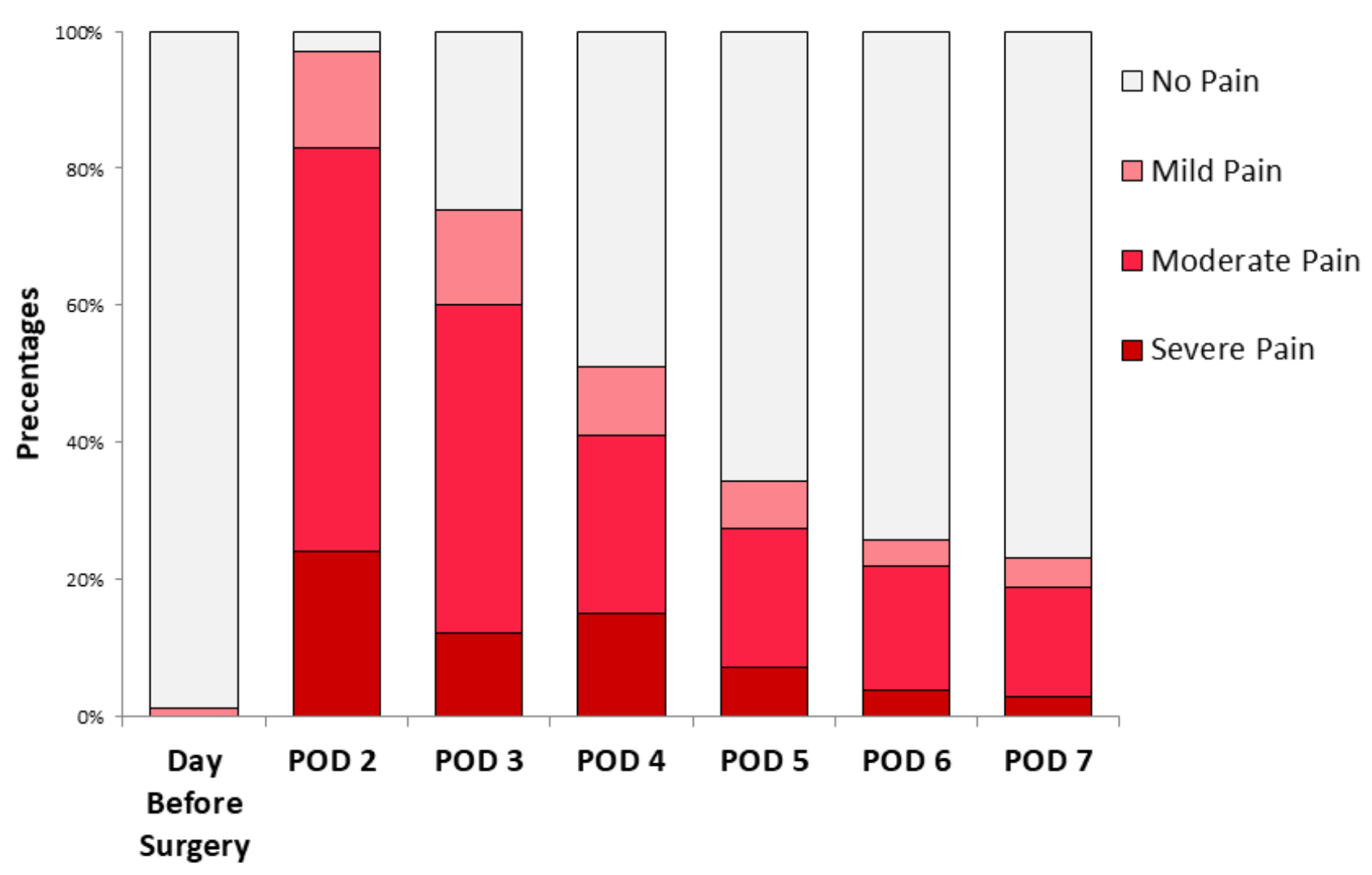

Figure 1

Perioperative Percentage of Patients with Severe, Moderate, and Mild Pain Intensity POD, post-operative day 


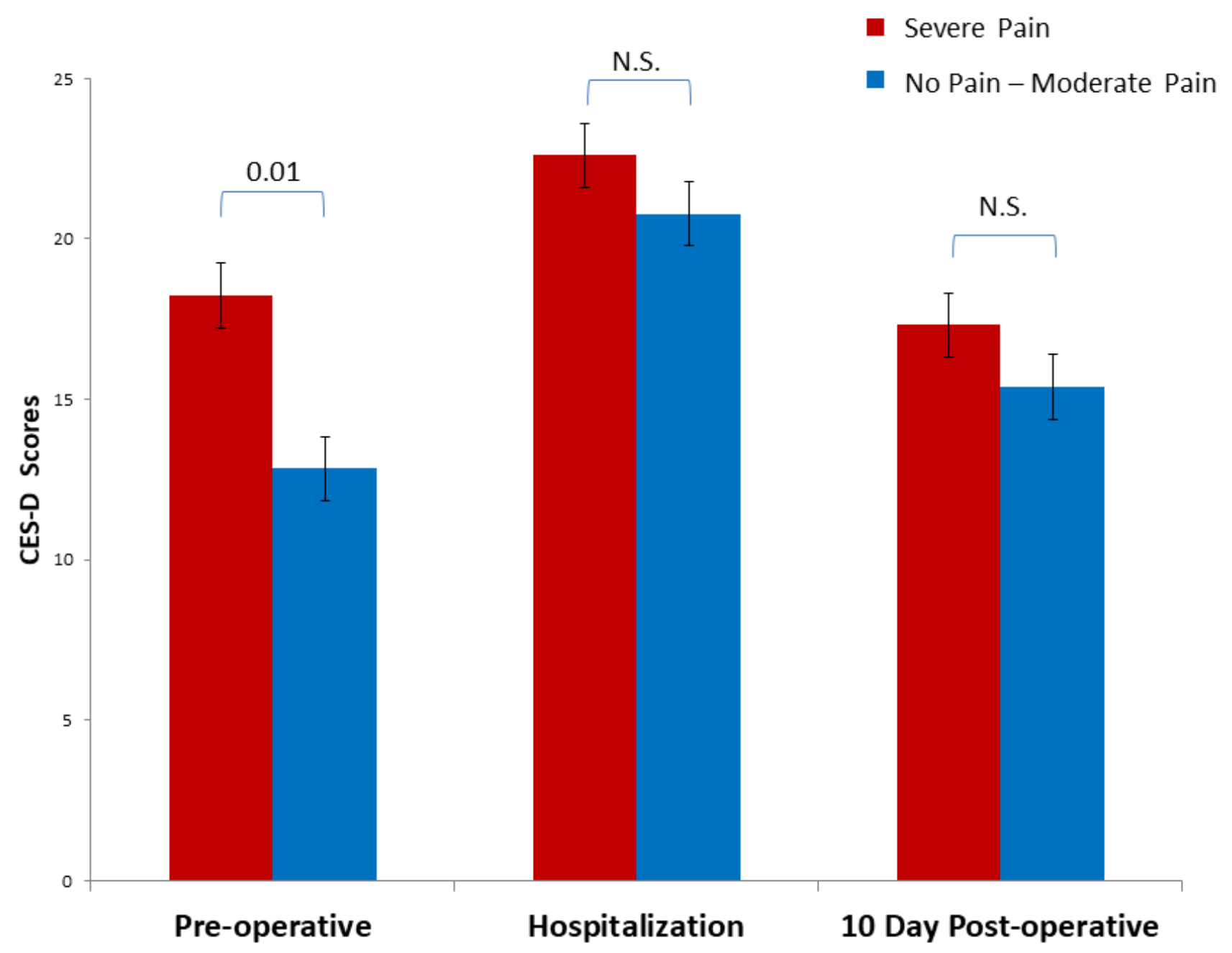

Figure 2

CES-D Scores at Preoperative, Hospitalization, and 10 days Post-operative Time Points in Post-Surgery Patients with Severe Pain versus Patients with No Pain to Moderate Pain Severity Red bars represent severe pain category. Blue bars represent no pain to moderate pain intensity category. $Y$ axis represents the Center for Epidemiological Study of Depression (CES-D) scores. 


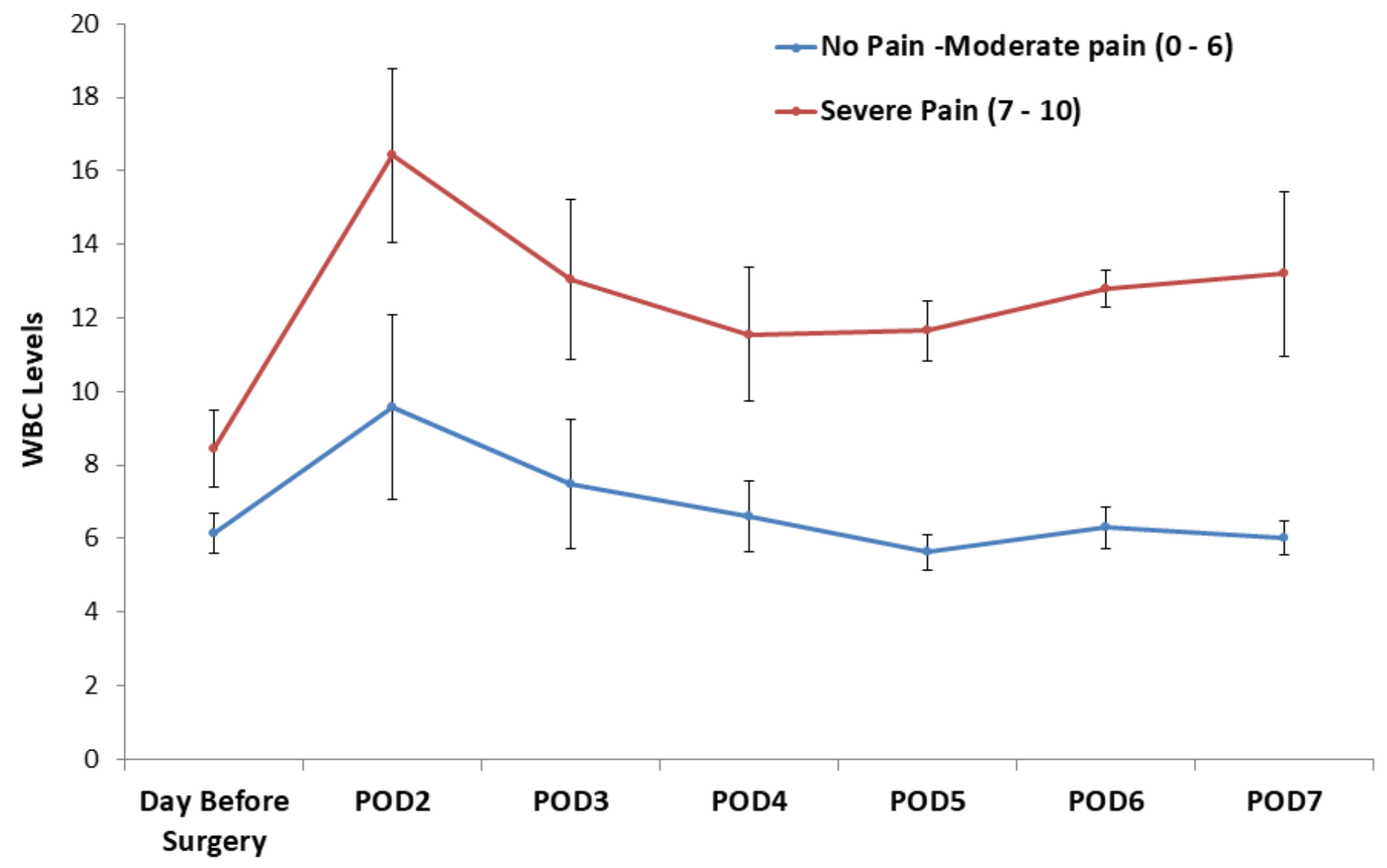

Figure 3

WBC Levels during Perioperative Period Red line represents severe pain category. Blue line represents no pain to moderate pain categories. $Y$ axis represents white blood Cells levels (WBC). POD, post-operative day 